\title{
Change in fractional anisotropy during treatment of diabetic ketoacidosis in children
}

\author{
Leslie Dervan', Andrew Poliakov ${ }^{2,3}$, Seth D. Friedman ${ }^{2,3}$, Dennis Shaw ${ }^{2-4}$, Catherine Pihoker ${ }^{1,3,5}$, Joan S. Roberts ${ }^{1,3}$, \\ Todd Richards ${ }^{4}$, Kenneth Marro ${ }^{4}$ and Monica S. Vavilala ${ }^{1,2,6}$
}

BACKGROUND: The pathophysiology resulting in cerebral edema in pediatric diabetic ketoacidosis (DKA) is unknown. To investigate the changes in white matter microstructure in this disease, we measured diffusion tensor imaging (DTI) parameters, including apparent diffusion coefficient (ADC), fractional anisotropy (FA), and radial and axial diffusivity in children with DKA at two time points during treatment.

METHODS: A prospective observational study was conducted at Seattle Children's Hospital, Seattle, WA. Thirty-two children admitted with DKA ( $\mathrm{pH}<7.3$, bicarbonate $<15 \mathrm{mEq} / \mathrm{l}$, glucose $>300 \mathrm{mg} / \mathrm{dl}$, and ketosis; $11.9 \pm 3.2 \mathrm{y}$; and $47 \%$ male) were enrolled and underwent two serial paired diffusion magnetic resonance imaging (MRI) scans following hospital admission. Seventeen of the 32 participants had diffusion tensor images of adequate quality for tract-based spatial statistics (TBSS) analysis.

RESULTS: TBSS mapping demonstrated main white matter tract areas with a significant increase in FA and areas with a significant decrease in ADC, from the first to the second MRI. Both radial and axial diffusivity terms showed change, with a diffuse pattern of involvement.

CONCLUSION: Consistent DTI changes occurred during DKA treatment over a short time frame. These findings describe widespread water diffusion abnormalities in DKA, supporting an association between clinical illness and DTI markers of microstructural change in white matter.

D iabetic ketoacidosis (DKA) is a common acute complication of diabetes in children, occurring in $15-67 \%$ of the patients with new-onset diabetes (1), with an average of 8 episodes per 100 patient-years in children with an existing diagnosis (2). DKA episodes are associated with the development of cerebral edema, which occurs at an estimated rate of 0.5-1 per 100 DKA episodes and carries a significant mortality, estimated at $21-23 \%(1,3,4)$. Prior research has suggested singular or combined roles for osmolar effects related to treatment (1), cerebral ischemia due to dehydration and hypocapnia $(3,5)$, and relative cerebral hyperemia, due to increased cerebral blood flow, impaired cerebral autoregulation, and/ or blood-brain barrier permeability (6-8). However, the specific pathophysiological processes in pediatric DKA that might contribute to cerebral edema are not well characterized.

Magnetic resonance imaging (MRI) is an important research tool which has documented gray and white matter changes in many neurological disease states, including DKA $(5,7,9,10)$. Diffusion-weighted MRI, by using magnetic field gradients to spatially label water molecules, reflects changes in water diffusion in tissues and is therefore considered sensitive to changes in the cerebral microstructure (11). Diffusion tensor imaging (DTI) measurements are sensitive to both absolute water diffusion and the direction of diffusion; for example, movement of water in normal pressure hydrocephalus from the white matter extracellular space to the intraventricular space is associated with a decrease in the apparent diffusion coefficient (ADC) (12). The fractional anisotropy (FA) changes in a wide variety of neurological disease states have been described $(9,10)$. However, there is, to date, no description of the FA change in DKA treatment. White matter disease typically causes a decrease in the directional mobility of water, decreasing the FA (9). Pathological changes are then often conceptualized based on FA features. For example, demyelination is associated with an increase in the radial (short-axis) term, whereas axonal damage is associated with a decrease along the axial (longaxis) direction $(10,11)$. In our prior work, extensive changes in FA were observed in a mitochondrial disease sample, including widespread reduction of FA in the white matter tracts, with an increase in the radial term and no change in the axial term (13). While acquisition parameters can impact the specificity of radial or axial terms (14), the general pattern of change has the potential to be informative about underlying mechanisms.

To add to our understanding of the mechanisms of cerebral edema, we aimed to describe regional patterns of DTI parameter changes during acute pediatric DKA treatment in this small study. We hypothesized that a vasogenic process would cause a relative increase in the extracellular fluid compartment within the brain during illness, leading to an increased radial diffusivity and a lower FA within white matter tracts during illness. We secondarily hypothesized that patients with clinical

\footnotetext{
'Department of Pediatrics, University of Washington, Seattle, Washington; ${ }^{2}$ Department of Radiology, Seattle Children's Hospital, Seattle, Washington; ${ }^{3}$ Center for Clinical and Translational Research, Seattle Children's Hospital, Seattle, Washington; ${ }^{4}$ Department of Radiology, University of Washington, Seattle, Washington; ${ }^{5}$ Division of Endocrinology and Diabetes, Seattle Children's Hospital, Seattle, Washington; ${ }^{6}$ Department of Anesthesiology, University of Washington, Seattle, Washington. Correspondence: Monica S. Vavilala (vavilala@uw.edu) 
evidence of cerebral edema would have lower FA during illness compared to patients without cerebral edema.

\section{RESULTS}

\section{Demographic and Clinical Characteristics}

Detailed clinical characteristics of 17 subjects are shown in Table 1. Mean age of subjects was 11.9 y (range: 6-18 y); 8 were male. For seven of the subjects, the episode of DKA was their initial clinical presentation of type 1 diabetes. Fifteen subjects were admitted to the pediatric intensive care unit and two were admitted to the inpatient ward service. All subjects were treated with intravenous (i.v.) insulin infusion $(0.1 \mathrm{U} / \mathrm{kg} / \mathrm{h})$ and received hydration with i.v. fluids for DKA before MRI scanning. Eleven subjects underwent head computed tomography (CT) at the discretion of the treating physician; five of which reported cerebral edema or increased intracranial pressure; four of these patients received mannitol. One additional patient, whose head CT was read as normal, received mannitol for symptoms consistent with cerebral edema. The three groups showed a difference in $\mathrm{pH}$ and bicarbonate that approached significance $(\mathrm{F}(2,14)=3.57$; $P=0.056$ and $\mathrm{F}(2,14)=3.12 ; P=0.076)$. No other demographic or admission clinical characteristics differed between the clinical groups.

We compared demographic and clinical variables between the subjects who constituted the final sample of the study $(n=17)$ and those who were excluded from the study $(n=27)$ because of failure to complete two MRIs $(n=12)$ or because of inadequate image quality $(n=15)$. The mean presenting glucose was higher among those included compared to those excluded (mean: 635 vs. $512 \mathrm{mg} / \mathrm{dl} ; P=0.041$ ). The presenting creatinine was also higher among those included compared to those excluded (mean: 1.19 vs. $0.94 \mathrm{mg} / \mathrm{dl} ; P=0.05$ ). Among those able to complete both MRIs, however, no difference in creatinine was present between those included and those excluded. There were no differences in the remainder of the variables evaluated, including presenting $\mathrm{pH}, \mathrm{pCO}_{2}$, bicarbonate, blood urea nitrogen, gender, age, and presence of a new diagnosis of diabetes between the two groups (Table 2).

\section{DTI and FA Data}

Average time to the first MRI (Time 1) was $19.1 \pm 7.7 \mathrm{~h}$. Average time to the second MRI (Time 2) was $42.6 \pm 12.2 \mathrm{~h}$. Average time elapsed between MRI from Time 1 to Time 2 was $23.4 \pm 11.9 \mathrm{~h}$. The tract-based spatial statistics (TBSS) DTI results are shown in Figure 1. Standardized white matter tracts are shown in green, with areas of statistically significant DTI parameter change, including FA, ADC, and radial $(\lambda \mathrm{r})$ and axial $(\lambda \mathrm{a})$ diffusivities, shown in blue (lower during illness, at Time 1) and yellow/red (higher during illness, at Time 1). There was a change in mean FA from Time 1 to Time 2, with 14 of 17 subjects (82\%) showing an increase in FA (Figure 2; $z=-2.44$; $P=0.015)$.

We did not observe any statistically significant association between either initial FA or FA change and age, gender, $\mathrm{pH}$, $\mathrm{pCO}_{2}$, bicarbonate, or blood urea nitrogen $(P \geq 0.1$ for all analyses). For the three groupings of subjects based on clinical severity, FA increased from Time 1 to Time 2 by $2.8 \%$ (group A), $1.7 \%$ (group B), and $4.3 \%$ (group C). The difference in initial FA among the three groups was not significant (Figure 3; $P=0.16)$.

The following two outliers were observed with respect to mean FA change (Figure 2, numerical data not shown): one patient with an increase over time and one with a decrease over time of $>2$ SDs from the mean FA change. The patient with the notable increase in FA from Time 1 to Time 2 had severe neurologic depression and received multiple doses of mannitol, tracheal intubation, and invasive intracranial pressure monitoring. This patient required follow-up for persistent neurological symptoms after discharge, with left-sided extremity weakness that resolved by 1 mo and persistent neuropathic pain of the left lower extremity. The patient with the notable decrease in FA from Time 1 to Time 2 had severe acidosis and dehydration, with a $\mathrm{pH}$ of 6.63 on presentation to the study institution, after having received initial i.v. fluid resuscitation; he received an additional $50 \mathrm{ml} / \mathrm{kg}$ in i.v. fluid resuscitation at the study institution. He had a head CT that was reported as normal, and he did not receive mannitol.

Table 1. Admission clinical characteristics of 17 children with diabetic ketoacidosis

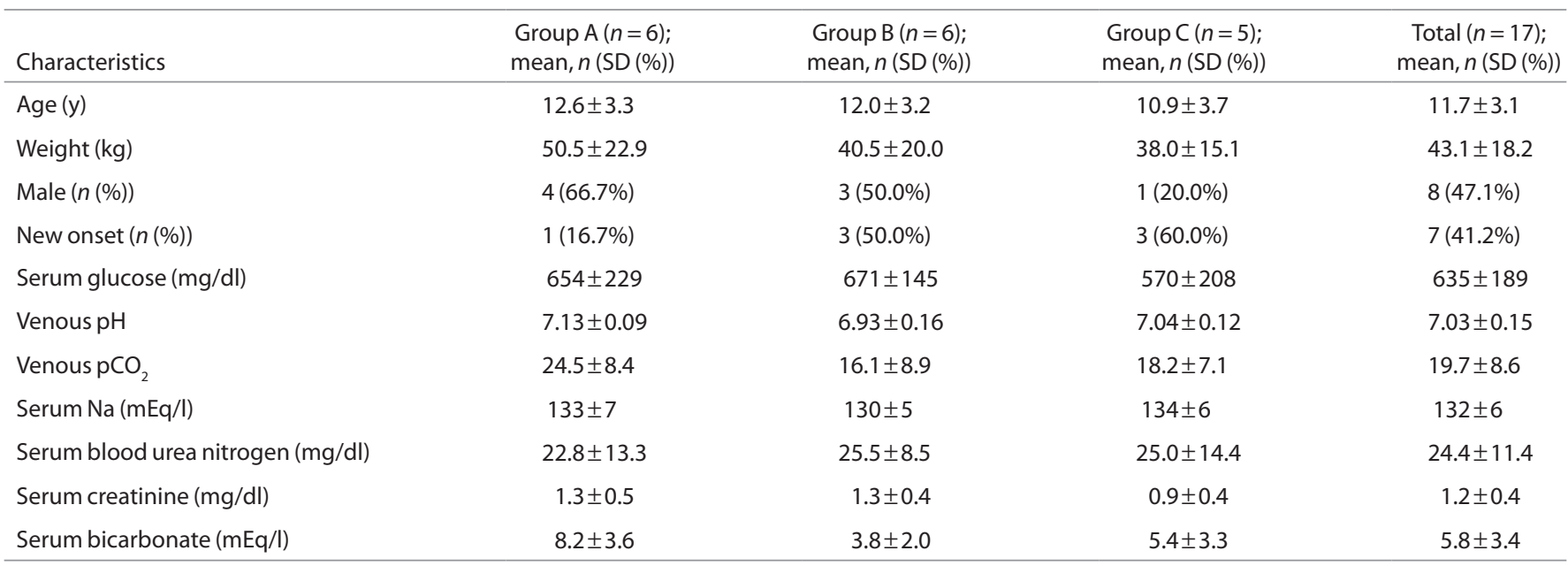


Table 2. Admission clinical characteristics of 44 children with diabetic ketoacidosis evaluated for inclusion

\begin{tabular}{|c|c|c|}
\hline Characteristics & $\begin{array}{c}\text { Included } \\
(n=17) ; \text { mean, } \\
n(\mathrm{SD}(\%))\end{array}$ & $\begin{array}{c}\text { Excluded } \\
(n=27) ; \text { mean, } \\
n(\mathrm{SD}(\%))\end{array}$ \\
\hline Age (y) & $11.7 \pm 3.1$ & $9.8 \pm 5.3$ \\
\hline Weight (kg) & $43.1 \pm 18.2$ & $40.3 \pm 22.6$ \\
\hline Male $(n(\%))$ & $8(47.1 \%)$ & $15(55.6 \%)$ \\
\hline New onset ( $n(\%))$ & $7(41.2 \%)$ & $18(66.7 \%)$ \\
\hline Serum glucose $(\mathrm{mg} / \mathrm{dl})^{\mathrm{a}}$ & $635 \pm 189$ & $512 \pm 188$ \\
\hline Venous pH & $7.03 \pm 0.15$ & $7.06 \pm 0.13$ \\
\hline Venous $\mathrm{pCO}_{2}$ & $19.7 \pm 8.6$ & $22.1 \pm 7.8$ \\
\hline Serum $\mathrm{Na}(\mathrm{mEq} / \mathrm{l})$ & $132 \pm 6$ & $136 \pm 7$ \\
\hline Serum blood urea nitrogen (mg/dl) & $24.4 \pm 11.4$ & $18.8 \pm 11.9$ \\
\hline Serum creatinine $(\mathrm{mg} / \mathrm{dl})^{\mathrm{a}}$ & $1.2 \pm 0.4$ & $0.9 \pm 0.4$ \\
\hline Serum bicarbonate $(\mathrm{mEq} / \mathrm{l})$ & $5.8 \pm 3.4$ & $7.3 \pm 4.4$ \\
\hline
\end{tabular}

${ }^{a}$ Average value differed between the two groups with $P \leq 0.05$.

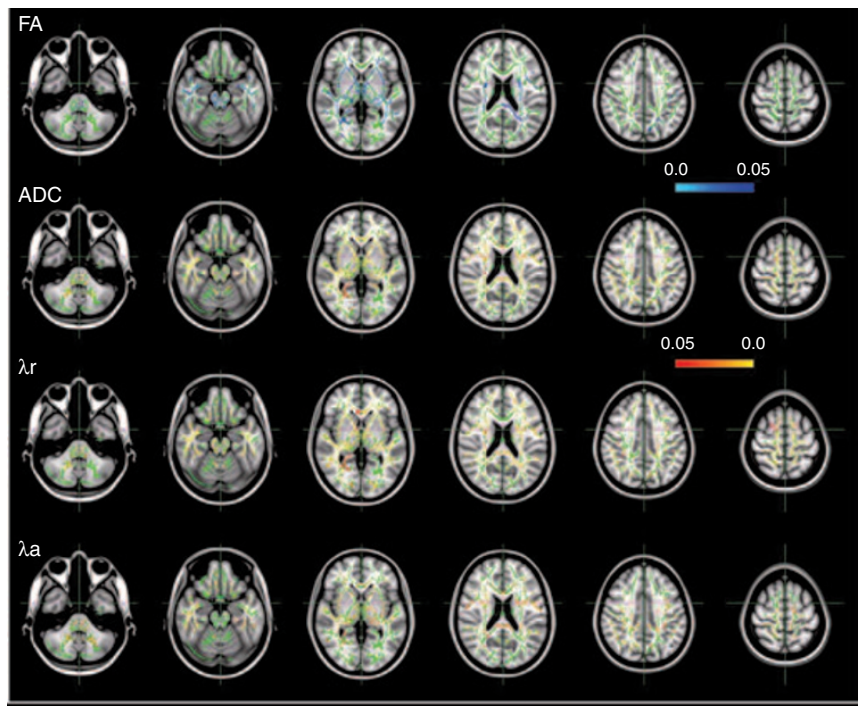

Figure 1. Areas of statistically significant diffusion tensor imaging parameter changes from first to second magnetic resonance imaging (MRI). Representative slices from tract-based spatial statistics analysis, showing the main white matter tract map (green) and superimposed results of statistical analysis $(P<0.05)$ for each MRI parameter. Significant areas of increase (blue) and decrease (red/yellow) in each MRI parameter (fractional anisotropy (FA), apparent diffusion coefficient (ADC), and radial and axial diffusivity $(\lambda r$ and $\lambda a)$ ) from first to second MRI are shown.

\section{DISCUSSION}

In this study, we describe the widespread change in FA, ADC, and axial and radial diffusivity calculated from DTI parameters during DKA treatment. FA increased during the study period, whereas $\mathrm{ADC}$ and radial and axial diffusivity were decreased. Subjects who underwent head CT or who received mannitol did not have a significantly lower mean FA at Time 1. Although the precise mechanisms involved in the development of cerebral edema remain uncertain, the observation of an increase in FA and a decrease in radial and axial diffusivity over time

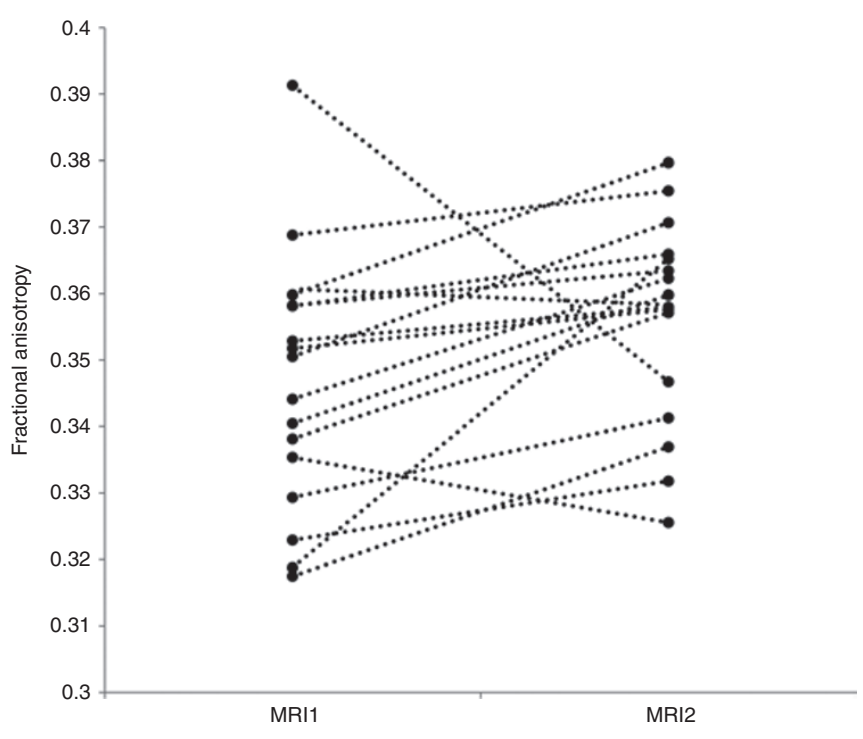

Figure 2. Change in mean white matter fractional anisotropy (FA) between Time 1 and Time 2 in 17 patients. Mean FA increased in 14 of 17 patients from Time 1 to Time $2(z=-2.44 ; P=0.015)$.

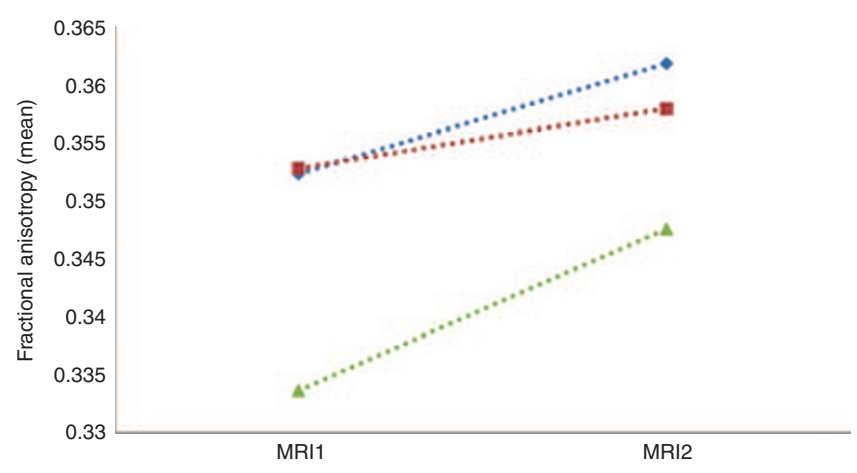

Figure 3. Stratified average mean white matter fractional anisotropy (FA) change from Time 1 to Time 2. The following groups of patients were compared: those who underwent no neuroimaging outside of the study protocol (group A; blue diamonds); those who underwent head computed tomography, at the discretion of the treating physician, but did not receive mannitol (group B; red squares); and those who received mannitol for presumed cerebral edema (group $C$; green triangles). Group A showed an average FA increase of $2.8 \%$. Group B, $1.7 \%$. Group C, $4.3 \%$. Average FA at Time 1 was $0.35 \pm 0.02,0.35 \pm 0.02$, and $0.33 \pm 0.01$ for groups $A, B$, and $C$, respectively $(P=0.16)$.

would be parsimoniously explained by vasogenic edema, present at first scan with resolution or improvement at follow-up. Results of this small study suggest the need for further investigation of the temporal changes in cerebral physiology in DKA.

Nearly all patients in this small study showed an increase in FA over time. This included patients who did not have recorded neurologic symptoms and is consistent with prior studies suggesting that even asymptomatic patients with DKA may have some level of cerebral edema (15). We did not observe an association with traditional markers of DKA severity (age, gender, $\mathrm{pH}, \mathrm{pCO}_{2}$, bicarbonate, and blood urea nitrogen) and either initial FA or FA change between Time 1 and Time 2. Although the power to detect such associations is limited in this small study, this may suggest that the observed 
MRI changes are independent of these clinical factors and support clinical observations that traditional markers of DKA severity often correlate poorly with the severity of neurologic symptoms. Further investigation is warranted to determine whether MRI parameters have better correlation than laboratory data with the symptoms of cerebral edema and whether these parameters could be used to better identify patients at risk of neurologic deterioration.

Other factors that could potentially influence the FA change over time remain important to consider; for example, acute ischemia leads to an elevated FA (10). In DKA, ischemia may play a role, secondary to dehydration and the cerebral effects of hypocarbia. In this study, the patient with the largest decrease over time in FA had extreme acidosis and hypocarbia on presentation and received a large volume of i.v. fluid resuscitation. Additionally, prior episodes of ischemia are associated with long-term lower FA values (creating the possibility of a lower "baseline" FA for some patients). All three subjects with a decrease over time in FA had a prior diagnosis of diabetes, and one had a prior episode of DKA-related cerebral edema, raising the possibility of an altered baseline in these patients.

Although the biochemical and neurological changes in DKA resolve rapidly, it is possible that the FA demonstrated by Time 2 in this study does not reflect a full return to baseline. "Normal" FA values vary significantly by scanning variables, age, and the specific white matter location studied. However, above the age of $3 \mathrm{y}$ and before age-related decline occurs, average FA values are reported between 0.45 and 0.6 for most main white matter tracts $(16,17)$. Although it is not possible to directly compare our FA values to published norms owing to different study protocols and scanners, our average FA values at Time 2 appear lower than these reported averages, suggesting persistent structural differences at Time 2.

We examined published data of FA and radial and axial diffusivity changes reported in other disease states to compare our findings to those in other mechanisms of cerebral edema. FA has been used extensively in the research setting to characterize central nervous system tumors, tumor infiltration, and peritumoral edema (18-20). Decreased FA is common in white matter injury, typically attributed to its association with an increased radial diffusivity $(\lambda \mathrm{r})$; this is observed in periventricular white matter in hydrocephalus, posterior reversible encephalopathy syndrome, peritumoral edema (10), and mitochondrial disease (13). A few disease states are reported in which a decreased FA is associated with decreased axial diffusivity $(\lambda \mathrm{a})$, including radiation injury (21). However, we identified few disease states reported with an increased axial diffusivity, the pattern we observed in this study.

There are limitations to this study. First, this is a small singlecenter study, and results from these subjects may not be generalizable to all children with DKA. Second, we were unable to obtain comparison data from patients with diabetes who were not affected by DKA, limiting our ability to describe FA changes to only the population and time frame that we studied. Without having this group, we evaluated published control data to help interpret the magnitude of changes observed. For the TBSS data of 8 - to 20 -year-old normative subjects, FA values increase on average by 0.002 per year (22). By contrast, our subjects demonstrated an FA change $>0.05$ (Figure 3), supporting the presence of a marked treatment-induced effect. Third, in our attempt to group by a measure of severity of neurologic symptoms, only some patients had head CT scans, at the discretion of the attending physician, precluding an analysis between imaging modalities. From a DTI perspective, there are imaging factors that introduce inherent variability in the data such as image noise, artifact, partial volume averaging between tissues in adjacent image slices, and regions of crossing white matter fibers (10). Although study variability is minimized somewhat by evaluating within-subject differences, further work at higher field and with greater resolution will be helpful to understand the radial and axial overlap. Although consistent results were observed in this sample, the small sample size and heterogeneity of clinical severity limited our ability to assess subgroup differences in response or baseline DTI parameters.

In summary, this study describes DTI changes including FA during pediatric DKA treatment. The observation that presumed FA and ADC abnormalities at baseline normalized with treatment correlates with clinical improvement. The notion that these directional water changes were related to both axial and radial terms may be informative to the underlying mechanism of cerebral edema, as few other disease states are described with this combination of findings. Further work will be instructive to refine and extend these results.

\section{METHODS}

After obtaining institutional approval from the Seattle Children's Hospital Human Subjects Review Committee, we conducted a prospective cohort study of 45 children admitted with DKA. Consent was obtained from their parents or legal guardians, and assent was obtained from patients per institutional protocol. From these 45 subjects enrolled, 1 subject withdrew consent, and 12 were unable to complete two serial MRI scans. Of the 32 subjects completing both the MRI scans, 17 had MRI data with diffusion tensor images that were of acceptable quality for TBSS mapping and FA calculation. Data from these subjects are reported here.

\section{Study Participants}

Patients to be admitted to Seattle Children's Hospital with a diagnosis of DKA were identified, approached by the clinical team, had consent and assent obtained (when applicable), and were prospectively enrolled in the emergency department, pediatric intensive care unit, or inpatient ward. Eligibility criteria included the following: age $\leq 17$ $\mathrm{y}$, venous $\mathrm{pH} \leq 7.3$, blood glucose $>300 \mathrm{mg} / \mathrm{dl}$, and bicarbonate $<15$ $\mathrm{mEq} / \mathrm{l}$, with positive urinary ketones. We excluded patients deemed to have hemodynamic instability by the treating physician and patients with no available parents/guardians for consent at the time of enrollment. We reviewed medical records for eligibility, relevant medical history, and physiological data. Thirty-two subjects completed two serial MRI scans. MRI scans were reviewed by two study radiologists, and only those who had two diffusion tensor images of acceptable quality for TBSS mapping and FA calculation were included in this study (17 subjects).

\section{MRI Acquisition During Illness and Recovery}

Study participants underwent MRI examinations if they were deemed hemodynamically stable by the treating physician and were capable of undergoing the MRI without sedation. No sedation was used in obtaining MRI scans. The timing of imaging was defined in relation to the start of continuous i.v. insulin therapy (Time 0). Illness 
was defined as the first $12-24 \mathrm{~h}$ from Time 0 , and we aimed to obtain the first MRI (Time 1) during this period. Recovery was defined as 36-72h from Time 0 , and we aimed to obtain the second MRI (Time 2) during this period. Additionally, we aimed to have at least 12-h elapse between serial MRI scans for each patient. Imaging was performed on Allegra 1.5T scanner (Siemens, Erlangen, Germany) using phased array coils. Standard clinical imaging included a highresolution sagittal T1 magnetization-prepared rapid acquisition of gradient echo, axial T2, and axial fluid attenuated inversion recovery. DTI used a single-shot echoplanar imaging sequence, with the following imaging parameters: TR/TE, $5800 / 96 \mathrm{~ms}$; number of signal intensityintensity averages: $1-2$. The $b$ values for diffusion weighting were 0 and $1,000 \mathrm{~s} / \mathrm{mm}^{2}$, with 10 gradient-encoding directions. Data were obtained with a matrix size of $128 \times 128$ and $1.8 \times 1.8 \mathrm{~mm}$ in-plane resolution. Section thickness was $3.5 \mathrm{~mm}$, with 40 sections covering the entire brain.

\section{Statistical Analysis}

The clinical characteristics of all patients enrolled in this study are presented using descriptive statistics. Data are presented as mean (SD) and $n(\%)$. We assessed for differences in demographic and clinical variables between those included and those excluded due to incomplete MRI acquisition or inadequate MRI quality, using Student's $t$-test. We assessed for associations between initial FA or FA change and age, gender, $\mathrm{pH}, \mathrm{pCO}_{2}$, bicarbonate, and blood urea nitrogen using $t$-test or linear regression as appropriate. As a proxy for clinical severity of cerebral edema, we grouped the patients into the following three clinical categories: no neuroimaging outside of the study protocol (group A); head CT, at the discretion of the treating physician but no administration of mannitol (group B); and treatment with mannitol for presumed cerebral edema (group C). The clinical characteristics of each group were compared using descriptive statistics and one-way ANOVA.

Voxelwise statistical analysis of the FA, ADC and axial and radial diffusivity data was carried out using TBSS, an approach that registers subjects data and limits permutation statistical comparisons to the anatomy corresponding to the fundamental tract skeleton. TBSS is part of the FSL software library, version 4.1, which was used for DTI data processing (Analysis Group, FMRIB, Oxford, UK).

To evaluate a single severity measure, the mean FA, calculated as an average for the entire white matter skeleton from the TBSS results, was obtained for each patient at Time 1 and Time 2. Although this metric includes both significantly changing and unchanging regions, it was considered the best a priori proxy for whole brain severity. Groups were compared at baseline (Kruskal-Wallis equality-ofpopulations rank test) and for change across the treatment interval (Wilcoxon signed-rank test). Data are presented as mean (SD), and $P<0.05$ reflects significance.

\section{STATEMENT OF FINANCIAL SUPPORT}

Funded by National Institute of Child Health and Human Development (grant 5R21HD049832-02), National Institutes of Health, Bethesda, MD.

Disclosure: There are no conflicts of interest.

\section{REFERENCES}

1. Lawrence SE, Cummings EA, Gaboury I, Daneman D. Population-based study of incidence and risk factors for cerebral edema in pediatric diabetic ketoacidosis. J Pediatr 2005;146:688-92.

2. Rewers A, Chase HP, Mackenzie T, et al. Predictors of acute complications in children with type 1 diabetes. JAMA 2002;287:2511-8.

3. Glaser N, Barnett P, McCaslin I, et al.; Pediatric Emergency Medicine Collaborative Research Committee of the American Academy of Pediatrics.
Risk factors for cerebral edema in children with diabetic ketoacidosis. The Pediatric Emergency Medicine Collaborative Research Committee of the American Academy of Pediatrics. N Engl J Med 2001;344:264-9.

4. Edge JA, Hawkins MM, Winter DL, Dunger DB. The risk and outcome of cerebral oedema developing during diabetic ketoacidosis. Arch Dis Child 2001;85:16-22.

5. Glaser NS, Wootton-Gorges SL, Marcin JP, et al. Mechanism of cerebral edema in children with diabetic ketoacidosis. J Pediatr 2004;145:164-71.

6. Roberts JS, Vavilala MS, Schenkman KA, Shaw D, Martin LD, Lam AM. Cerebral hyperemia and impaired cerebral autoregulation associated with diabetic ketoacidosis in critically ill children. Crit Care Med 2006;34:221723.

7. Vavilala MS, Marro KI, Richards TL, et al. Change in mean transit time, apparent diffusion coefficient, and cerebral blood volume during pediatric diabetic ketoacidosis treatment. Pediatr Crit Care Med 2011;12:e344-9.

8. Vavilala MS, Richards TL, Roberts JS, et al. Change in blood-brain barrier permeability during pediatric diabetic ketoacidosis treatment. Pediatr Crit Care Med 2010;11:332-8.

9. Rollins NK. Clinical applications of diffusion tensor imaging and tractography in children. Pediatr Radiol 2007;37:769-80.

10. Alexander AL, Lee JE, Lazar M, Field AS. Diffusion tensor imaging of the brain. Neurotherapeutics 2007;4:316-29.

11. Neil JJ. Diffusion imaging concepts for clinicians. J Magn Reson Imaging 2008;27:1-7.

12. Demura K, Mase M, Miyati T, et al. Changes of fractional anisotropy and apparent diffusion coefficient in patients with idiopathic normal pressure hydrocephalus. Acta Neurochir Suppl 2012;113:29-32.

13. Ishak GE, Poliakov AV, Poliachik SL, et al. Tract-based spatial statistical analysis of diffusion tensor imaging in pediatric patients with mitochondrial disease: widespread reduction in fractional anisotropy of white matter tracts. AJNR Am J Neuroradiol 2012;33:1726-30.

14. Wheeler-Kingshott CA, Cercignani M. About "axial" and "radial" diffusivities. Magn Reson Med 2009;61:1255-60.

15. Glaser NS, Wootton-Gorges SL, Buonocore MH, et al. Frequency of subclinical cerebral edema in children with diabetic ketoacidosis. Pediatr Diabetes 2006;7:75-80.

16. Kochunov P, Williamson DE, Lancaster J, et al. Fractional anisotropy of water diffusion in cerebral white matter across the lifespan. Neurobiol Aging 2012;33:9-20.

17. Lebel C, Gee M, Camicioli R, Wieler M, Martin W, Beaulieu C. Diffusion tensor imaging of white matter tract evolution over the lifespan. Neuroimage 2012;60:340-52.

18. Bieza A, Krumina G. Magnetic resonance study on fractional anisotropy and neuronal metabolite ratios in peritumoral area of cerebral gliomas. Medicina (Kaunas) 2012;48:497-506.

19. De Belder FE, Oot AR, Van Hecke W, et al. Diffusion tensor imaging provides an insight into the microstructure of meningiomas, high-grade gliomas, and peritumoral edema. J Comput Assist Tomogr 2012;36:577-82.

20. Min ZG, Niu C, Rana N, Ji HM, Zhang M. Differentiation of pure vasogenic edema and tumor-infiltrated edema in patients with peritumoral edema by analyzing the relationship of axial and radial diffusivities on 3.0T MRI. Clin Neurol Neurosurg 2013;115:1366-70.

21. Wang S, Wu EX, Qiu D, Leung LH, Lau HF, Khong PL. Longitudinal diffusion tensor magnetic resonance imaging study of radiation-induced white matter damage in a rat model. Cancer Res 2009;69:1190-8.

22. Westlye LT, Walhovd KB, Dale AM, et al. Life-span changes of the human brain white matter: diffusion tensor imaging (DTI) and volumetry. Cereb Cortex 2010;20:2055-68. 